\title{
Esophageal Stenosis - a Notable Complication of Epidermolysis Bullosa - case report
}

\author{
Roxana-Mihaela Balcan*,, Irina-Liviana Stoian', Gabriela Paduraru, ${ }^{1,2}$, Ancuta Ignat', \\ Marin Burlea ${ }^{1,2}$ \\ 1 "Grigore T. Popa" University of Medicine and Pharmacy, lasi, ${ }^{2} \mathrm{~V}^{\text {th }}$ Gastroenterology Department, \\ "Sf. Maria" Emergency Hospital for Children, Iasi, Romania
}

\begin{abstract}
Epidermolysis bullosa is a rare condition caused by a genetic defect of the anchoring proteins between the epidermis and the dermis with an incidence of 1/50000. A 2 years and 7 months old, male infant was admitted in the Gastroenterology department of the pediatric hospital for severe dysphagia, the physical exam revealing skin lesions, scarring and nail abnormalities suggestive for Epidermolysis bullosa. The upper gastrointestinal endoscopy revealed a friable mucosa with ulcerative injuries and a severe esophageal stenosis. Epidemolysis bullosa, a condition involving mostly the skin can also be associated with severe mucosal injury that may cause serious medical complications.
\end{abstract}

Keywords: esophageal stenosis, Epidermolysis bullosa, mucosal lesions, blisters

\section{Introduction}

Epidermolysis Bullosa (EB) is a heterogeneous group of diseases characterized by a genetic defect of the anchoring proteins between the epidermis and the dermis and can be inherited either autosomal recessively or autosomal dominant. The disease manifests by the occurrence of blisters or erosions both on the surface of the skin and, in some cases, on the epithelial lining of the organs, as a response to minor trauma [1].

Received: August 2014; Accepted after review: September 2014; Published: September 2014

${ }^{*}$ Corresponding author: Roxana-Mihaela Balcan, "Grigore T. Popa" University of Medicine and Pharmacy, 16 Universitatii St., 700115, lasi, Romania. Email: apetroaei.roxana@hotmail.com
Based on the level of tissue separation within the cutaneous basement membrane zone there are three major types of EB: simplex, junctional and dystrophic, each of them caused by a genetic mutation and each of them having various subtypes that display individual clinical effects [2].

\section{Case report}

A two years and 7 month-old male infant was admitted in the $\mathrm{V}^{\text {th }}$ Gastroenterology Department, "Sf. Maria" Emergency Hospital for Children, lasi, on February 2013 for severe dysphagia for solids. The physical examination revealed erythematous squamous lesions located mostly on the hands and feet (Figure 1 and Figure 2) caused apparently by the rupture of blisters, and nail abnormalities. No respiratory or cardiovascular abnormalities were noted. The abdomen was not distended, 
the palpation revealed no tenderness, the liver and spleen edges were parallel to costal margins. The frequency and consistency of the stools were normal.

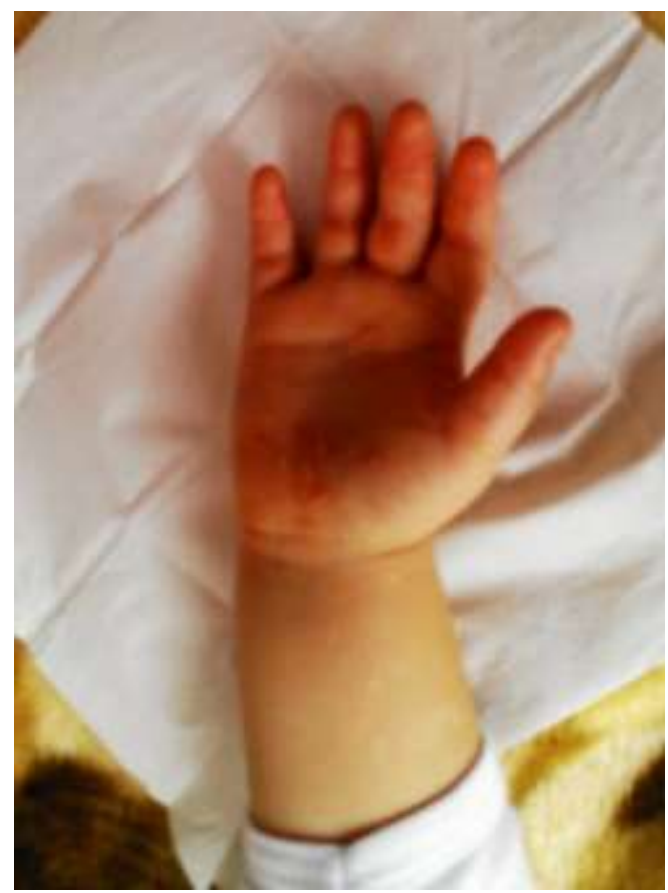

Fig. 1. Blisters and erythematous lesions located on the right hand

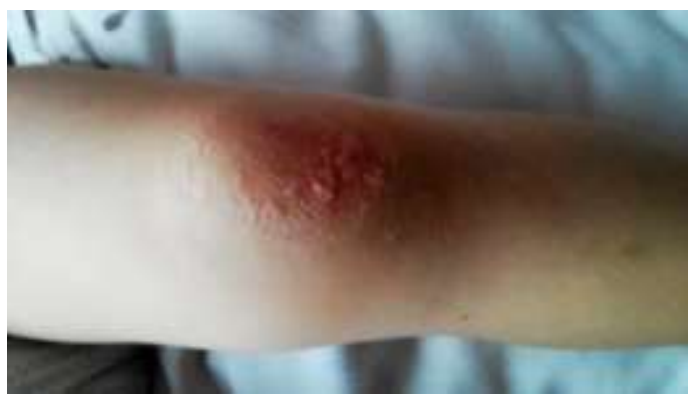

Fig. 2. Erythematous squamous lesion

Peripheral blood tests and a skin biopsy from lesion were performed. The full blood count showed an increased number of white blood cells and a low hemoglobin level.

The abdominal ultrasound indicated a liver normal in size, with diffuse hyperechogenic appearance, a contracted gallbladder and no renal abnormalities. An upper gastrointestinal endoscopy was performed and revealed a friable mucous membrane, with ulcerative injuries (Figure 3) and a severe esophageal stenosis at $20 \mathrm{~cm}$ from the incisors which could not be surpassed with the $9 \mathrm{~mm}$ endoscope.

Following these findings, the patient received pulse corticosteroid therapy, Proton pump inhibitors and prokinetics with a favorable result, the patient being able to resume oral ingestion of food.

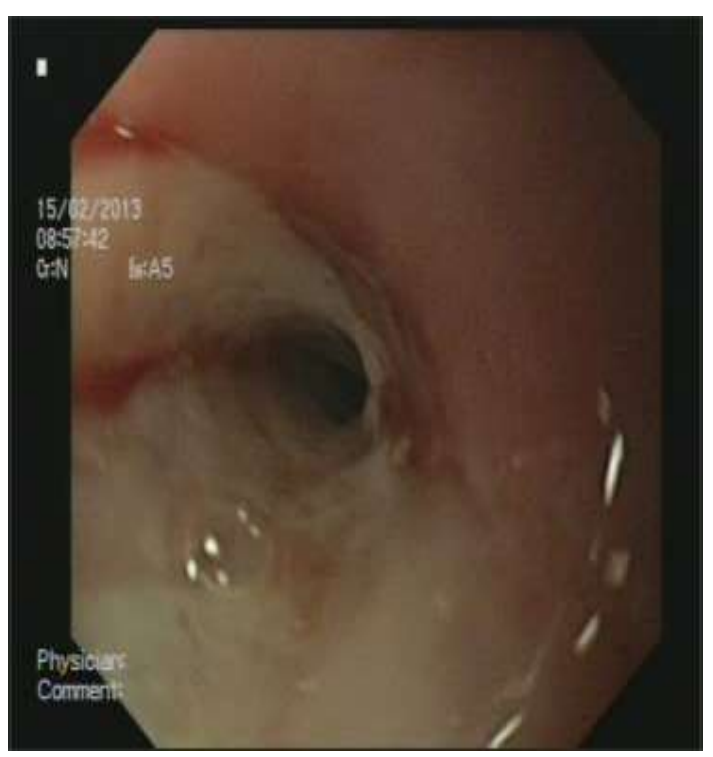

Fig. 3. Endoscopic appearance of the esophagus at the admission

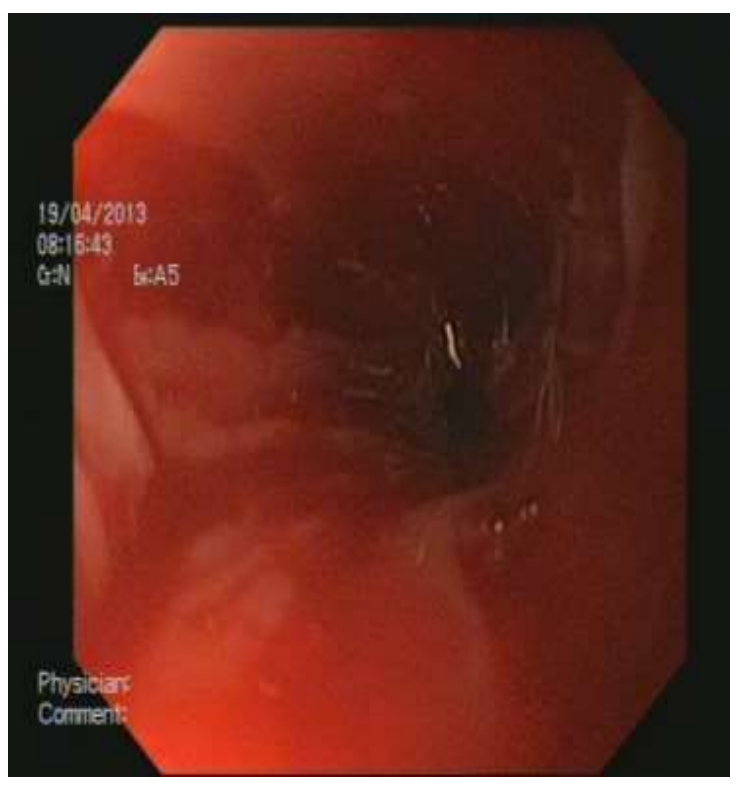

Fig. 4. Endoscopic appearance of the esophagus after two months 
At the follow up, two months later, the histopathology report confirmed the diagnosis of EB. The upper gastrointestinal endoscopy (Figure 4) was performed again and showed a friable mucosa, the esophageal stenosis but the absence of the ulcerations. An attempt to dilate the constricted area was performed with no success; however, the patient was in relatively good health and didn't address the hospital since.

The therapeutic guidelines for this patient are complex, and involve firstly the strong advice to avoid trauma, high temperature and humid environments which exacerbate the condition. In this particular case, the standard treatment for the esophageal involvement is the endoscopic balloon dilatation as the medical treatment is often ineffective [3].

\section{Discussion}

Epidermolysis bullosa is a rare condition, with a global incidence of 1 in 50000 births [1]. In Romania there are 72 documented families who are affected by this condition and, since 2012, there is a National Program for the treatment of these patients run by the Ministry of Health [4].

The disease is usually diagnosed in pediatric patients and in the absence of clear evidence and family history of EB there are a few differential diagnosis regarding the skin lesions which must be excluded such as: Staphylococcal scalded skin syndrome, bullous impetigo, chemical burns, drug or viralinduced toxic epidermal necrolysis and bullous mastocytosis [5].

Death in these patients occurs not due to the disease itself, but to its severe complications. The poorly treated skin lesions may generate non healing wounds that lead to sepsis, scarring, joint contracture and later on, squamous cell carcinoma [1].

Due to mucosal involvement, patients are also at risk for upper airway occlusion and failure to thrive as severe esophageal stenosis may impair ingestion. Because of the many clinical forms of EB, the therapeutic guidelines are complex and must be patient oriented and multi-disciplinary. They consist of general principles such as nutritional support, pain management and psychological evaluation and most specific guidelines, regarding the correct management of lesions. Wound care is a very important part of the treatment and it starts with correctly evaluating the severity, using an EB severity score. The care giver of the patient must periodically give baths using clorhexidine $0.1 \%$ or vinegar solutions in order to prevent infections especially before surgical procedures. Furthermore, the necrotic tissue must be debrided and dressings must be used to cover the wounds, dressings that changed periodically prevent further trauma and infections [4].

In regards to new therapies, there are a few ideas that researchers are currently looking into such as gene therapy, protein replacement therapy or bone marrow stem cell therapy [6]. There is high hope that in the future, with the proper use of old and new medical approach, the patients with EB will have a better chance to a normal life and in the end a higher survival rate.

\section{Conclusions}

Epidermolysis bullosa, a condition involving mostly the skin, can also be associated with severe mucosal injury that may cause serious medical complications more threatening to the patient's outcome than surface ones.

The diagnosis is based mostly on the physical examination and there is no available curative treatment, making the management of the patient a difficult task. Because of the high mortality rate of some types, such as Herlitz or letalis form of Junctional Epidermolysis Bullosa, which is close to $87 \%$ in the first year of life [7], the disease must be diagnosed early on and properly treated in order to avoid complications and improve the survival rate. 


\section{References}

1. Surasak P. Pediatric Epidermolysis Bullosa. Medscape Drugs \& Diseases, 2013, [http://emedicine.medscape.com/article/909549 -overview\#a0101 available at 9.09.2014]

2. Sawamura, Nakano H, Matsuzaki Y. Overview of Epidermolysis Bullosa. J Dermatol 2010; 37(3):214-219.

3. Karakan T, Dogan I, Cindoruk $M$, et al. Fluoroscopically guided endoluminal balloon dilation of oesophageal stricture due to dystrophic epidermolysis bullosa in two sisters. Acta Gastroenterol Belg 2006; 69(Suppl.03):327-329.

4. Salavastru M, Sprecher E, Panduru M, et al. Recommended strategies for epidermolysis bullosa management in Romania. MAEDICA J Clin Med 2013; 8(2):200-205.

5. Duijsters C, Halbertsma F, Kornelisse R, Arents $\mathrm{N}$, Andriessen P. Reccuring staphyloccocal scalded skin syndrome in a very low birth weight infant: a case report. Journal of Medical Case Reports 2009; 3:7313.

6. Hsu C, Wang SP, Yu-Yun Lee J, McGrath J. Treatment of Hereditary Epidermolysis Bullosa: Updates and Future Prospects. Am J Clin Dermato 2014; 15(1):1-6.

7. Mrinkovich P. Epidermolysis Bullosa. Medscape Drugs \& Diseases, 2013, [http://emedicine.medscape.com/article/106293 9-overview\#a0199 available at 9.09.2014]. 